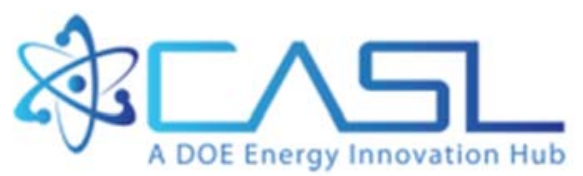

CASL-U-2016-1239-000

\title{
Canopy for VERAView Installation Guide
}

September 12, 2016
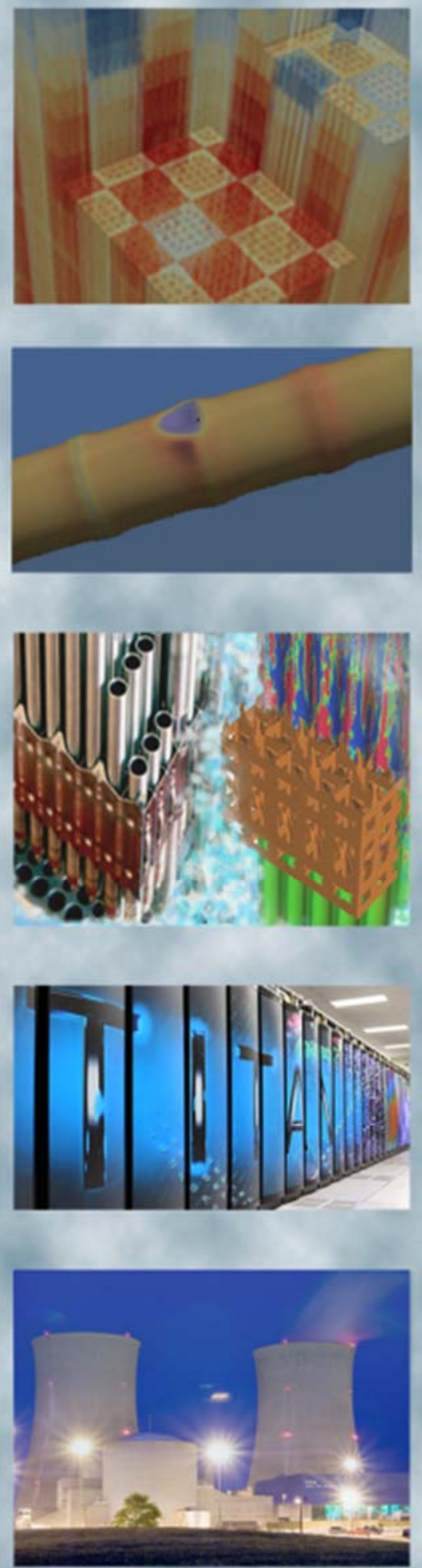


\section{REVISION LOG}

\begin{tabular}{|c|c|c|l|}
\hline Revision & Date & Affected Pages & \multicolumn{1}{c|}{ Revision Description } \\
\hline 0 & & All & Initial Version \\
\hline & & & \\
\hline & & & \\
\hline & & & \\
\hline
\end{tabular}

Document pages that are:

Export Controlled ____ None

IP/Proprietary/NDA Controlled None

Sensitive Controlled____ None

\section{Requested Distribution:}

To: Unlimited distribution

Copy: 


\title{
DOCUMENT AVAILABILITY
}

Reports produced after January 1, 1996, are generally available free via US Department of Energy (DOE) SciTech Connect.

Website http://www.osti.gov/scitech/

Reports produced before January 1, 1996, may be purchased by members of the public from the following source:

\author{
National Technical Information Service \\ 5285 Port Royal Road \\ Springfield, VA 22161 \\ Telephone 703-605-6000 (1-800-553-6847) \\ TDD 703-487-4639 \\ Fax 703-605-6900 \\ E-mail info@ntis.gov \\ Website http://www.ntis.gov/help/ordermethods.aspx
}

Reports are available to DOE employees, DOE contractors, Energy Technology Data Exchange representatives, and International Nuclear Information System representatives from the following source:

Office of Scientific and Technical Information

PO Box 62

Oak Ridge, TN 37831

Telephone 865-576-8401

Fax 865-576-5728

E-mail reports@osti.gov

Website http://www.osti.gov/contact.html

This report was prepared as an account of work sponsored by an agency of the United States Government. Neither the United States Government nor any agency thereof, nor any of their employees, makes any warranty, express or implied, or assumes any legal liability or responsibility for the accuracy, completeness, or usefulness of any information, apparatus, product, or process disclosed, or represents that its use would not infringe privately owned rights. Reference herein to any specific commercial product, process, or service by trade name, trademark, manufacturer, or otherwise, does not necessarily constitute or imply its endorsement, recommendation, or favoring by the United States Government or any agency thereof. The views and opinions of authors expressed herein do not necessarily state or reflect those of the United States Government or any agency thereof. 


\section{How to Install Canopy for VERAView}

\section{Overview}

With the addition of the 3D volume slicer widget, VERAView now relies on Mayavi and its dependents. Enthought's Canopy Python environment provides everything VERAView needs, and pre-built Canopy versions for Windows, Mac OSX, and Linux can be downloaded from:

https://store.enthought.com/downloads/\#default

However, after installing Canopy, it is necessary to use the Canopy Package Manager to install the $h 5 p y$, mayavi, and wxPython-3 modules VERAView needs. The steps required to install Canopy in order to run VERAView are described here for Windows, Mac OSX, and Linux.

\section{Note:}

Enthought is continuously updating Canopy. As of this version of this document, the latest Canopy version is 1.7.4. The screen shots in the instructions below were captured from an install of version 1.6.1. Whenever a newer version of a required package is available, use the latest available. If an error occurs when running VERAView, downgrade the wxPython version to 3.0.2.0-1.

\section{Windows}

\subsection{Step 1: Install Canopy}

Download canopy-1.7.4-win-64.msi from the Enthought site.:

https://store.enthought.com/downloads/\#default

Note you can download a 32-bit version from Enthought if needed.

Launch the installer by clicking or double-clicking (as per your Folder options) the file in Windows Explorer, typing the filename in Command Prompt, or some other method. Note this is a per-user install that does not require elevated privileges.

If you receive a security warning such as shown below, activate the Run button to continue the install.

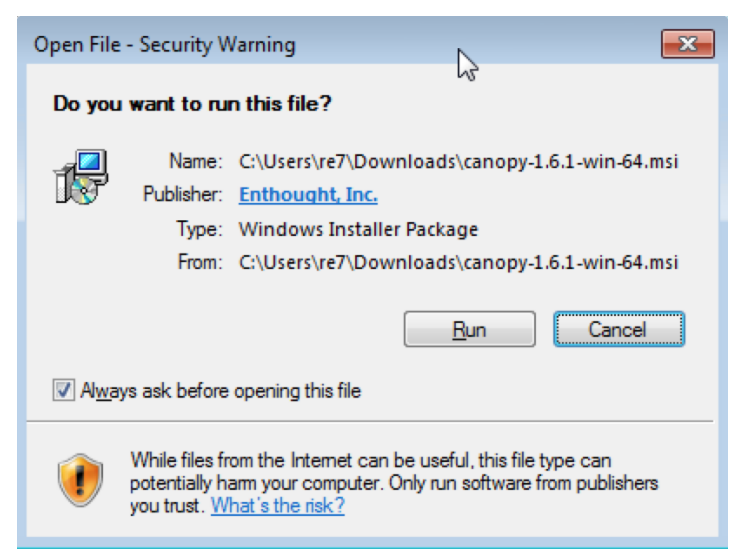


Activate the Next button when the Canopy installer wizard appears as shown below. Note you should use the default per-user install.

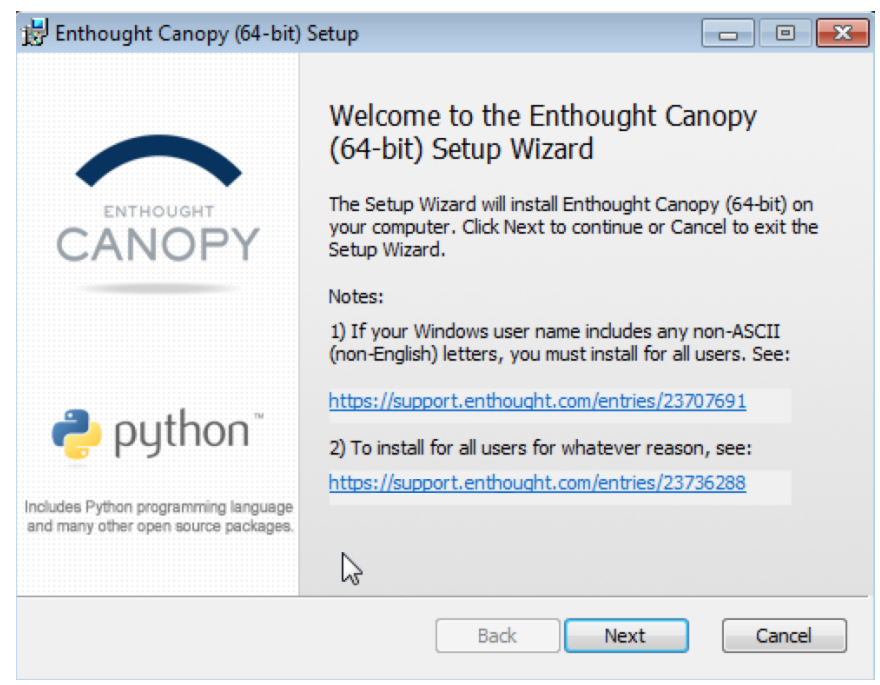

Accept the license and activate the Next button.

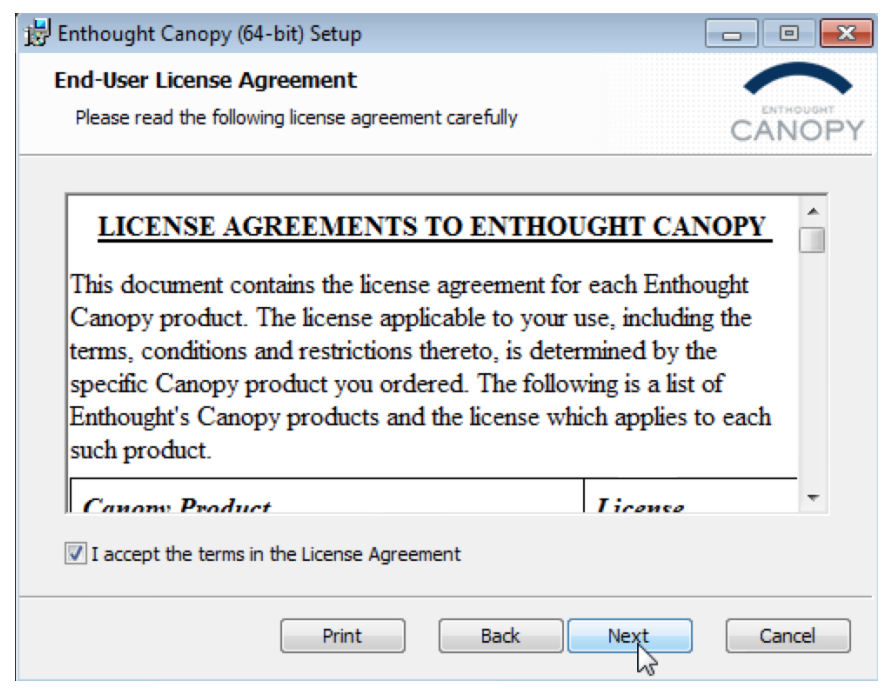

Continue the installation by activating the Next button after being informed of the per-user install and then activating the Install button. 


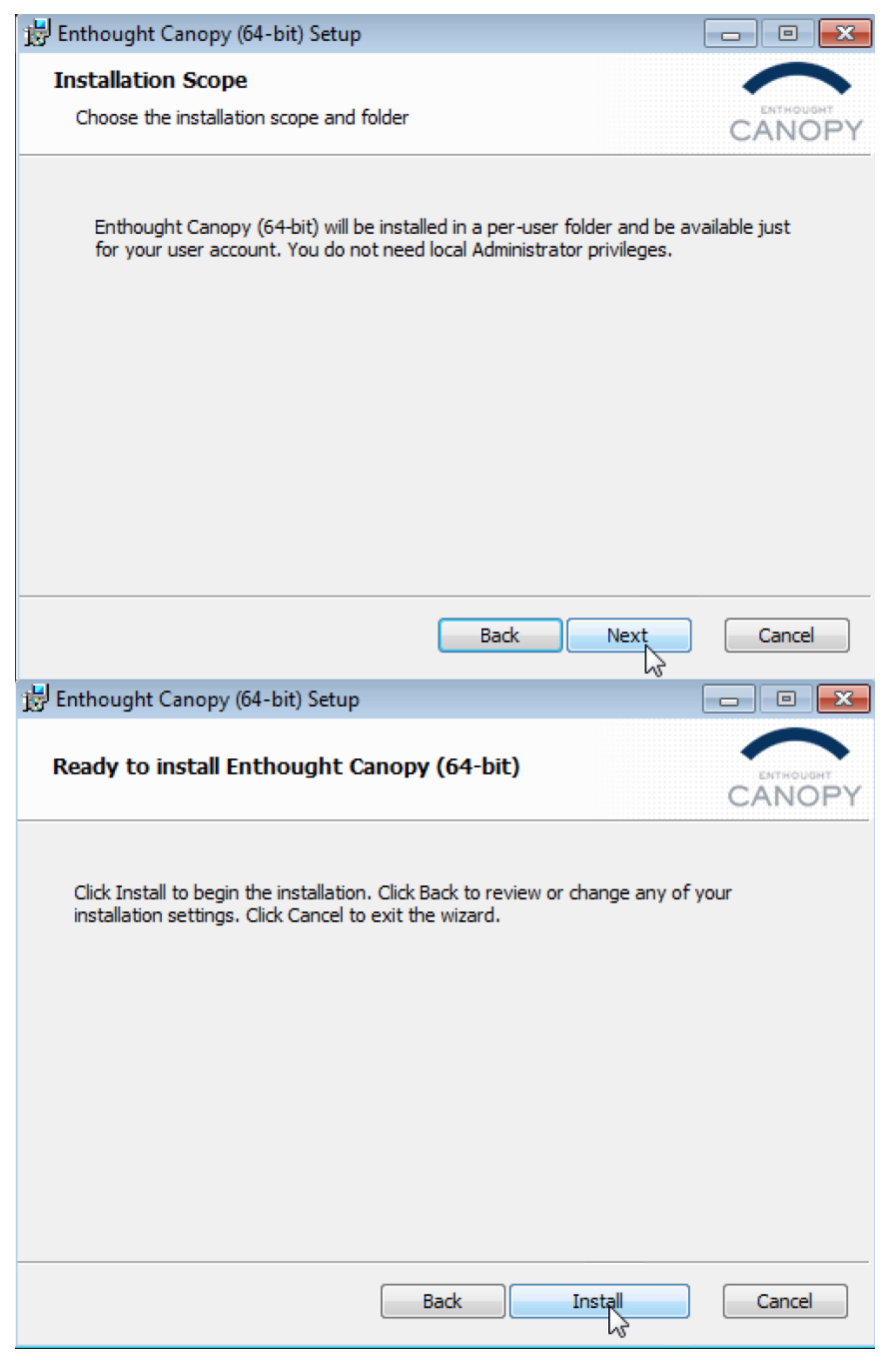

Complete the installation by checking Launch Canopy when setup exits and activating the Finish button.

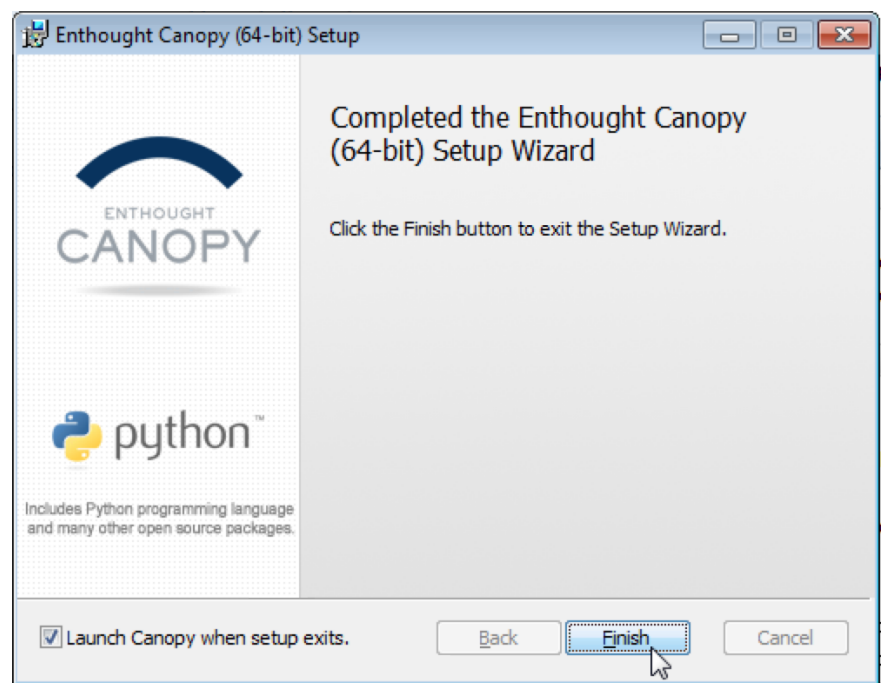




\subsection{Step 2: Setup the Canopy Environment}

Upon the first execution, the Canopy application will set up the environment for your user account. By default, Canopy will install to \%homepath\% \AppData \Local\Enthought \Canopy. If you change this location, you will need to manually edit the veraview.run.bat file inside your VERAView installation directory. Unless you have good reason to choose an alternate location (via the Change button), accept the default directory and activate the Continue button.

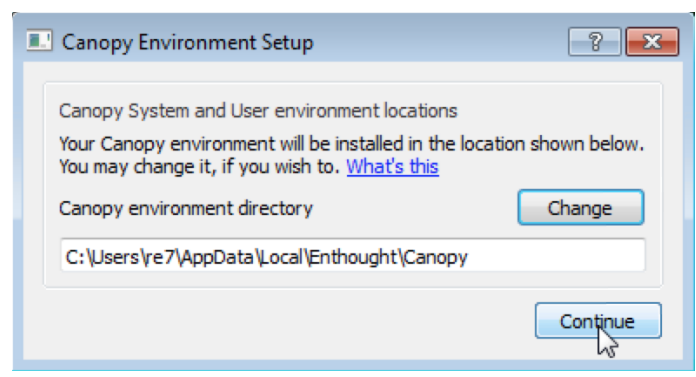

After a few seconds, a progress dialog will appear. It can take several minutes for the setup process to complete.

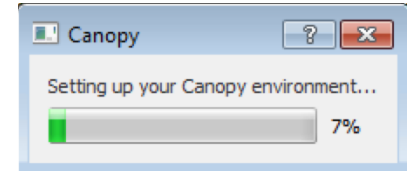

When the setup completes, you will be prompted to make Canopy's Python your default Python environment. This is completely up to you, for the VERAView launch script will reference Canopy directly. Activate the Starting using Canopy button to relaunch the Canopy application.

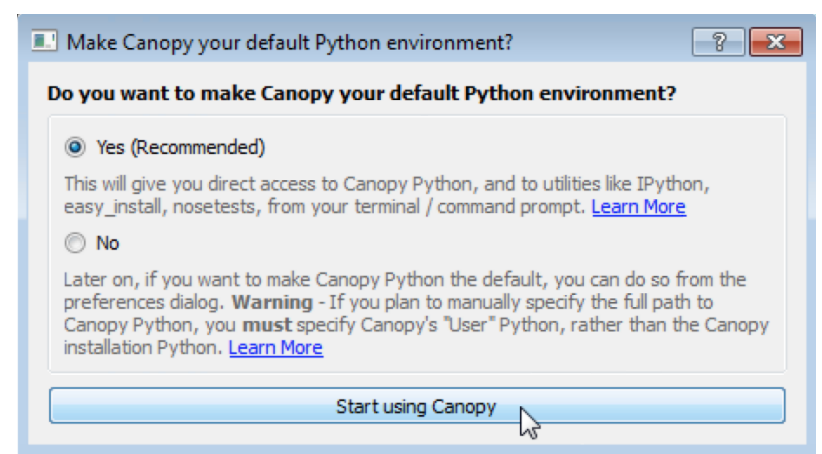

\subsection{Step 3: Install the h5py Module}

From the Canopy application, launch the Package Manager by clicking on its icon. 


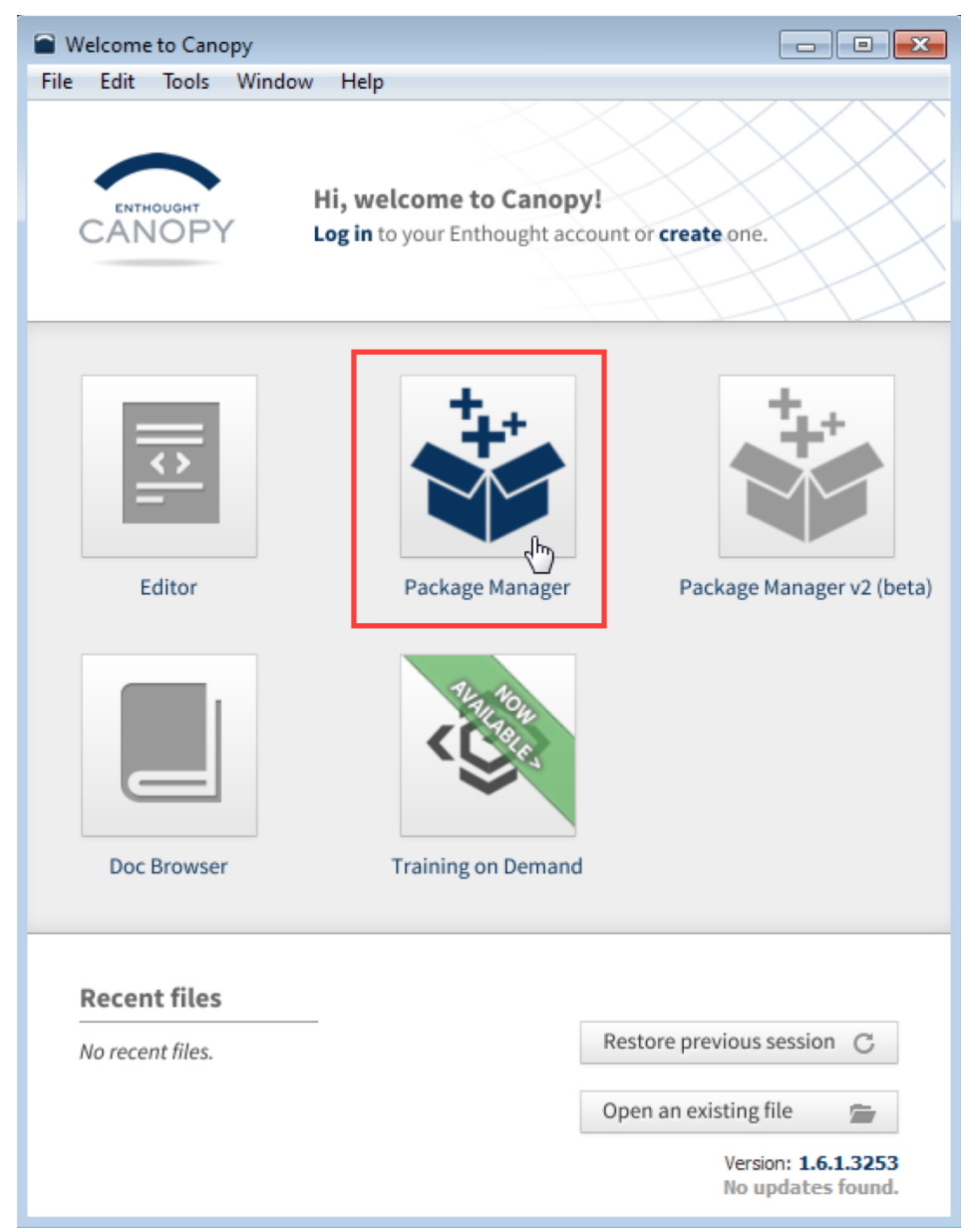

In Package Manager, select Available Packages selected and enter "h5py" in the search box. The package named "h5py 2.6.0-2" will appear in the package list panel. 


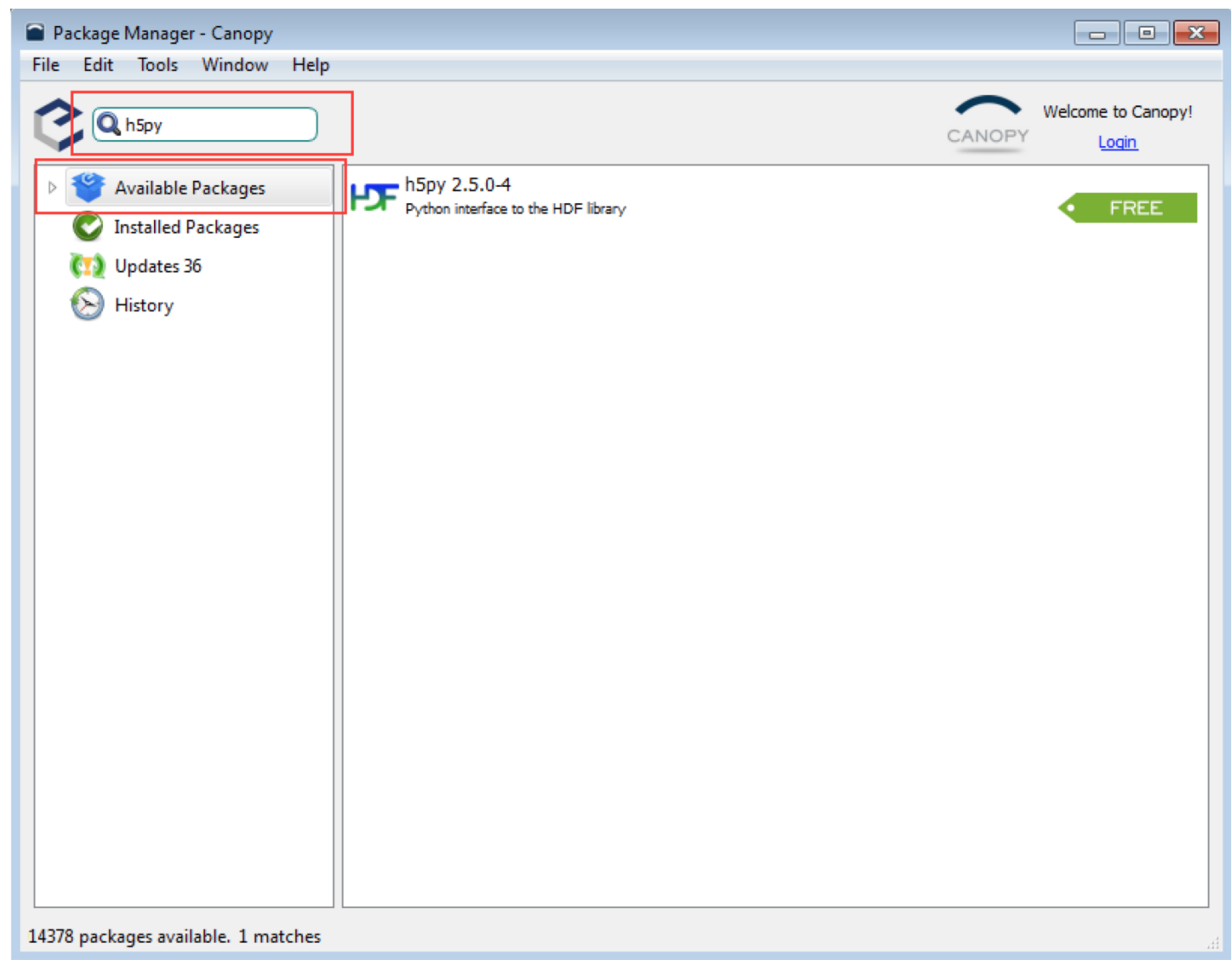

Click $h 5 p y$ 2.6.0-2 in the list panel to select it. Activate the Install (or Upgrade to) button to install it.

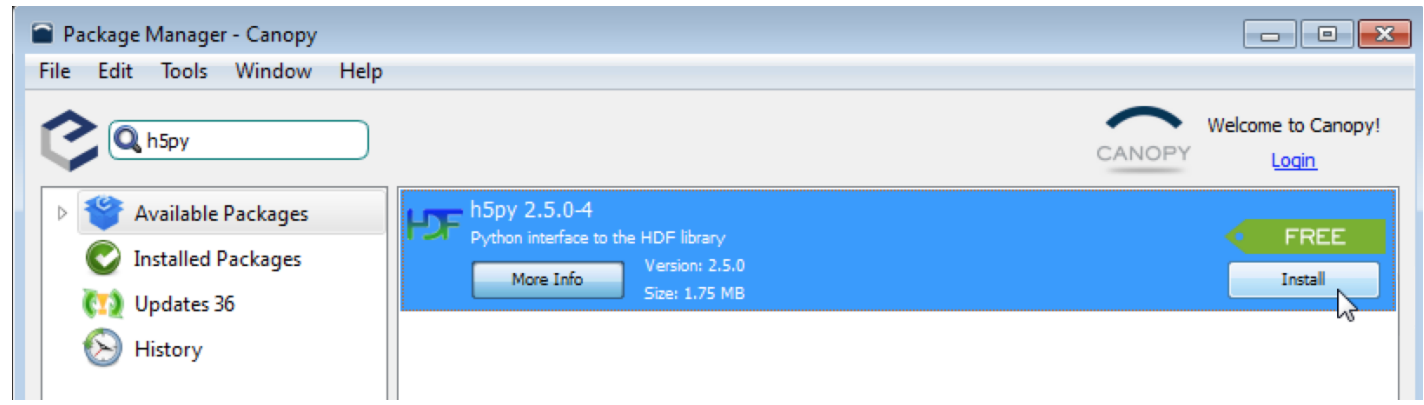

The module and dependency eggs will be downloaded and installed. When complete, the Install button will be renamed to Remove.

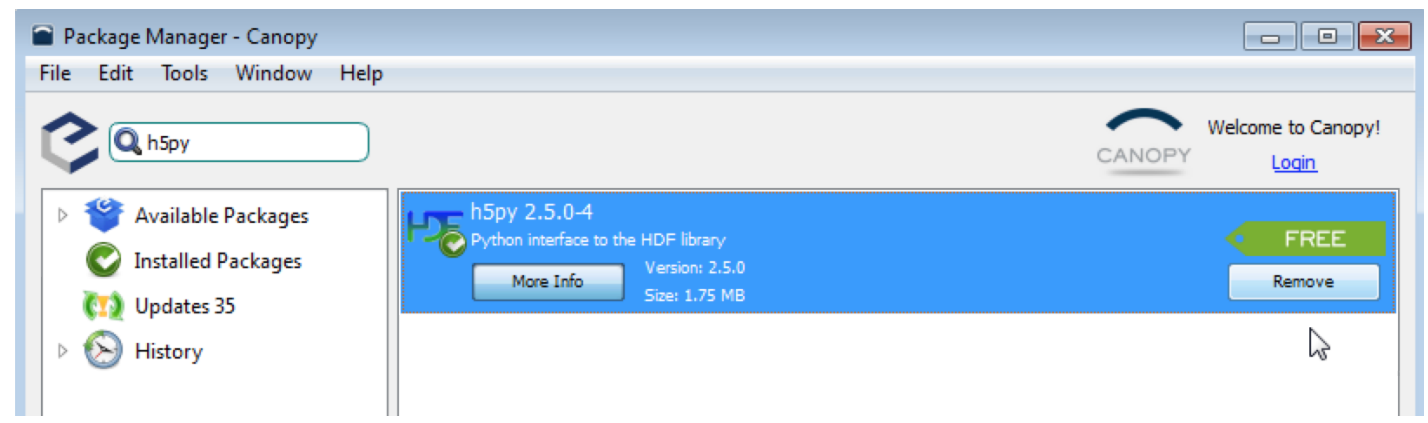




\subsection{Step 4: Install the wxPython-3.0.2 Module}

As per Step 3, seach for "wxPython" in Available Packages. This will result in wxPython 3.0.2.0-3 appearing in the list panel. Click wxPython 3.0.2.0-3 and activate the Install (or Upgrade to) button to install wxPython and all its dependencies.

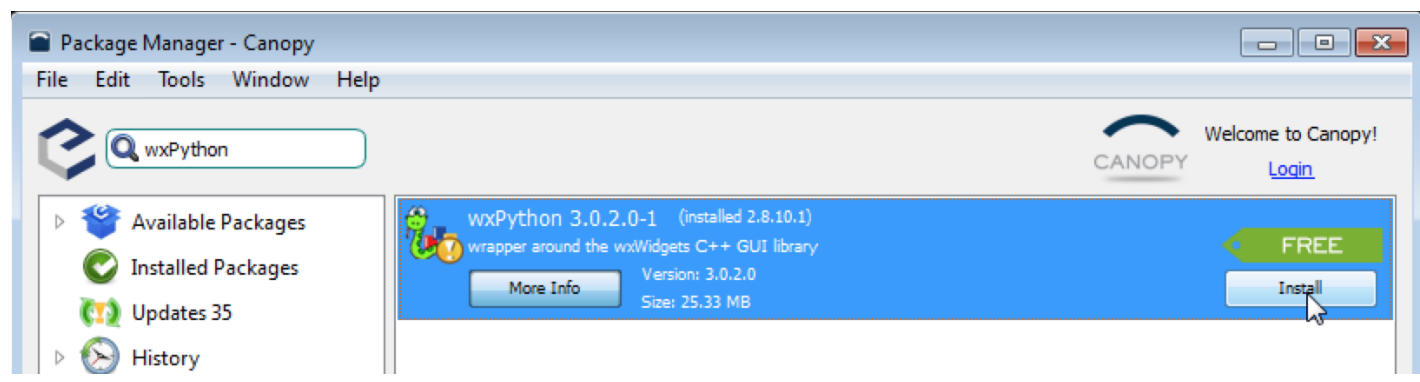

\section{Note:}

If VERAView fails with a message about image formats, you should downgrade to wxPython 3.0.2.0-01 by selected that version from the Available combo box and activating the Downgrade to v3.0.2.0-1 button.

\subsection{Step 5: Install the mayava-4.4.3 Module}

As per Steps 3 and 4, search for "mayavi" in Available Packages. This will result in mayavi 4.4.3-10 appearing in the panel. Click mayavi 4.4.3-10 and activate the Install (or Upgrade to) button.

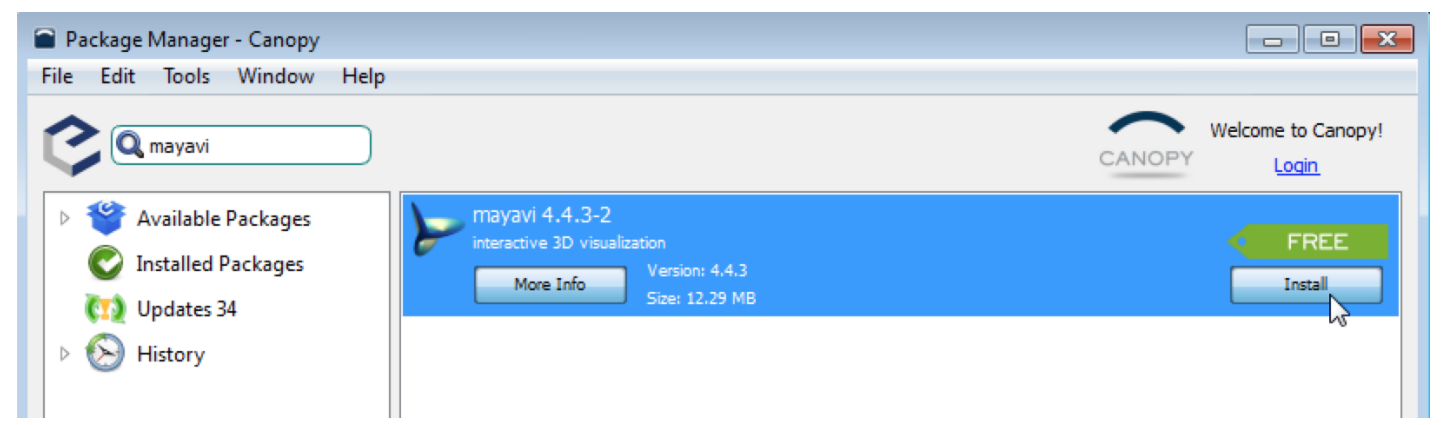

\subsection{Step 6: Test the Environment}

This is an optional but recommended step that involves launching a Command Prompt and running Python from the command line shell. The shell can be launched in one of two ways. First, using Windows Explorer locate the file \%userprofile\% $\backslash$ AppData \Local \Enthought \Canopy \User \python. exe and "open" it by clicking or double-clicking as per your Folder options. This should bring up an interactive prompt window. 


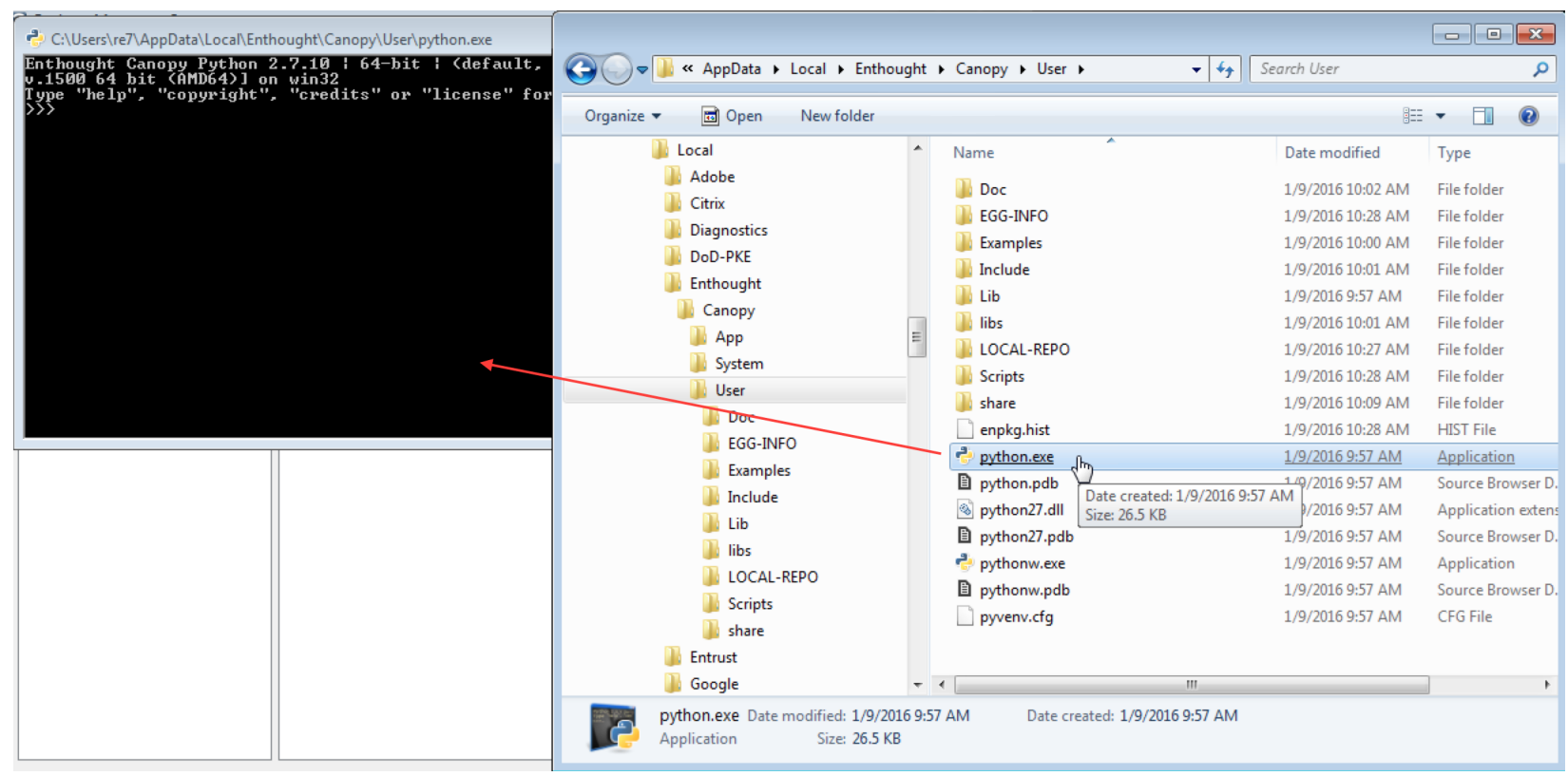

Second, open Command Prompt and execute the path above.

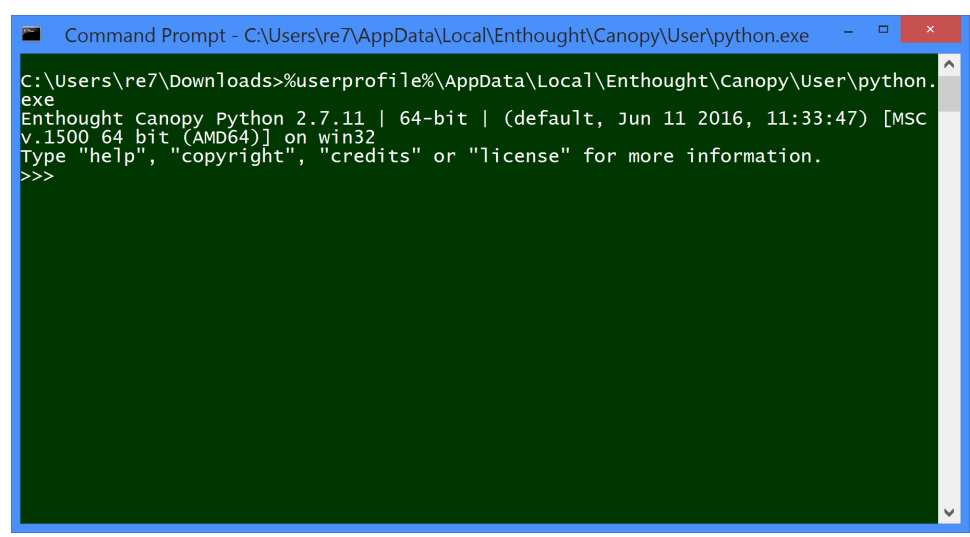

Download the following script to test that needed modules are available.:

https://casl:rocks@newton.ornl.gov/ re7/xfer/casl/test/test-install.py

Run the test script from the command line.:

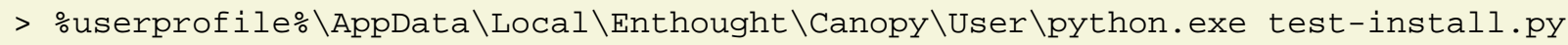
Importing h5py and wx...

wx.version $=3.0 .2 .0 \mathrm{msw}$ (classic)

Importing numpy...

Importing mayavi...

Good to go! 


\section{Mac OSX}

\subsection{Step 1: Install Canopy}

Download canopy-1 7.4-osx-64.dmg (or the latest version) from the Enthought site:

https://store. enthought.com/downloads/\#default

Open or mount the installer image by double-clicking it in Finder or using the open command in a Terminal bash shell:

\$ open canopy-1.7.4-osx-64.dmg

A Finder window will appear when the image is mounted.

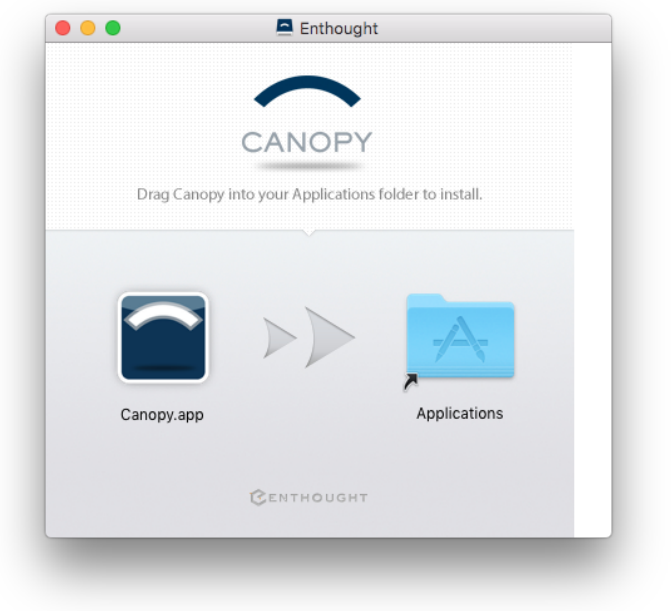

In the Finder window, drag the Canopy.app icon onto the Applications folder icon to initiate the install. A progress window will appear during the copy.

Note, if you are an Admin user, the install will copy the application into the root level

/Applications. Otherwise, it will be installed in /Applications.

When the install finishes, you can unmount the installer image by clicking the unmount/eject icon in the main Finder window (under "Devices") or by using the diskutil command in a Terminal window:

\$ diskutil unmount /Volumes/Enthought 


\subsection{Step 2: Run the Canopy Application}

Run the Canopy application by double-clicking the Canopy.app icon under Applications in the Finder.

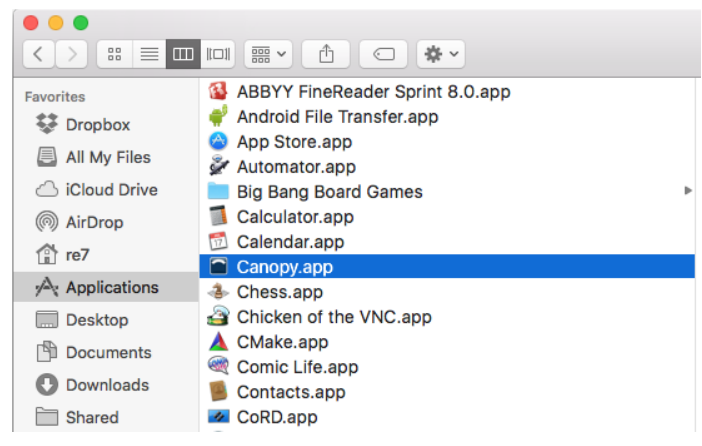

Depending on your Security \& Privacy configuration, you may be presented with a security challenge since Canopy is not a signed application.

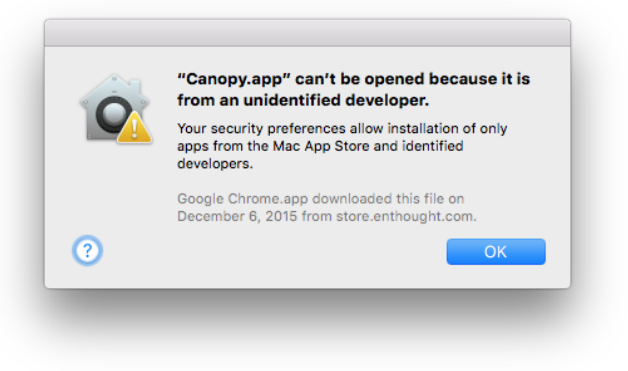

If so, click $O K$ to remove the dialog. Return to the Finder window. Instead of double-clicking the Canopy.app icon, type and hold the <Control> key while left-clicking Canopy.app and choose Open from the popup menu. This will bring up another dialog verifying you want to run Canopy. Click Open on that dialog.

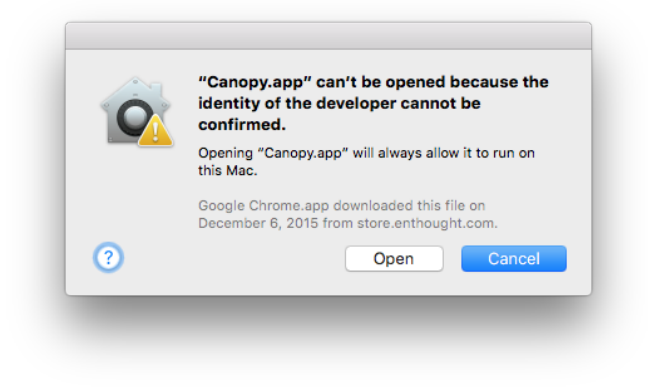

If for some reason this does not work, it may be necessary to open System Preferences from Apple menu. Click Security \& Privacy. 


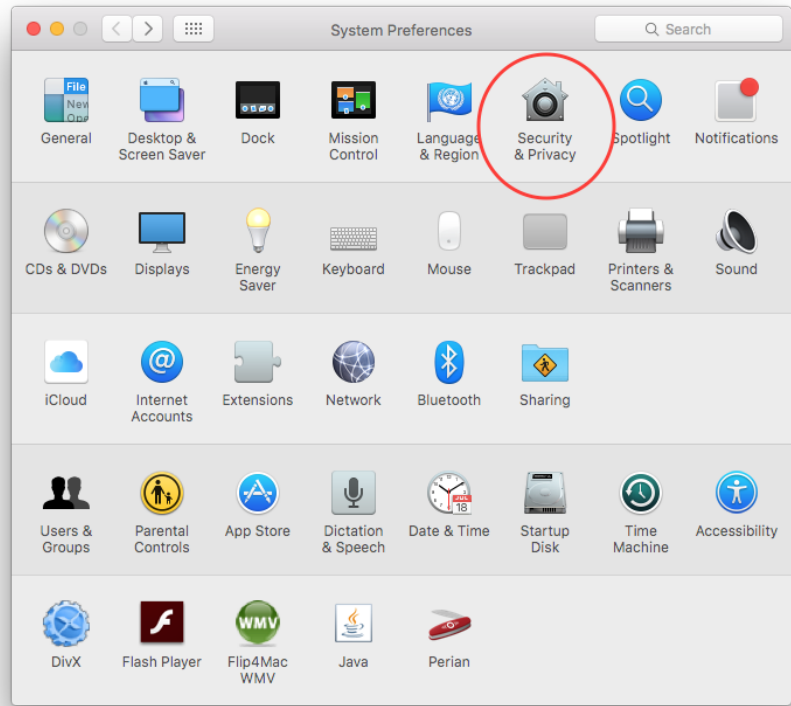

Select the General tab. If necessary, click the lock icon to make changes.

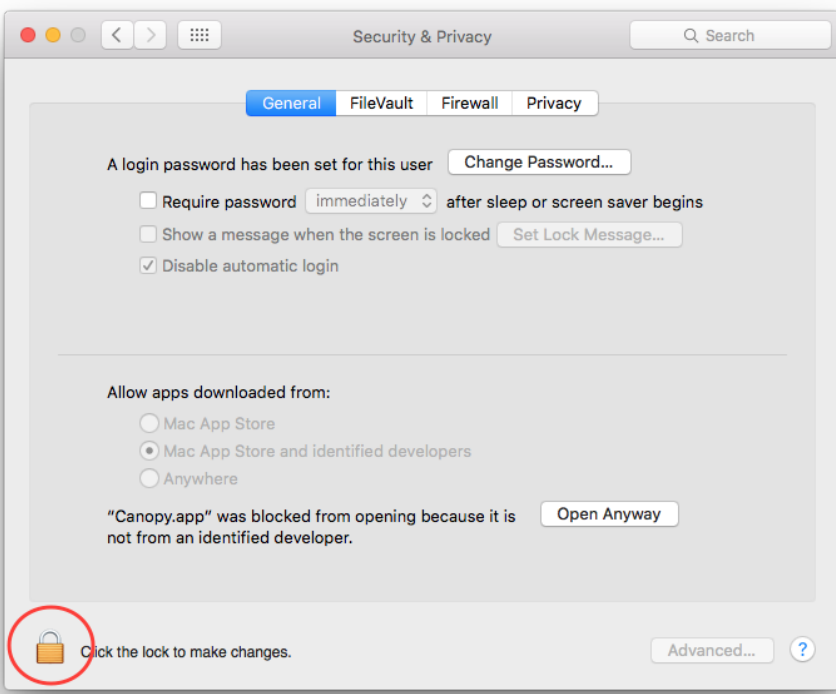

At least temporarily, click the Anywhere radio button under *Allow apps downloaded from. you may restore your original Allow apps downloaded from settings. 


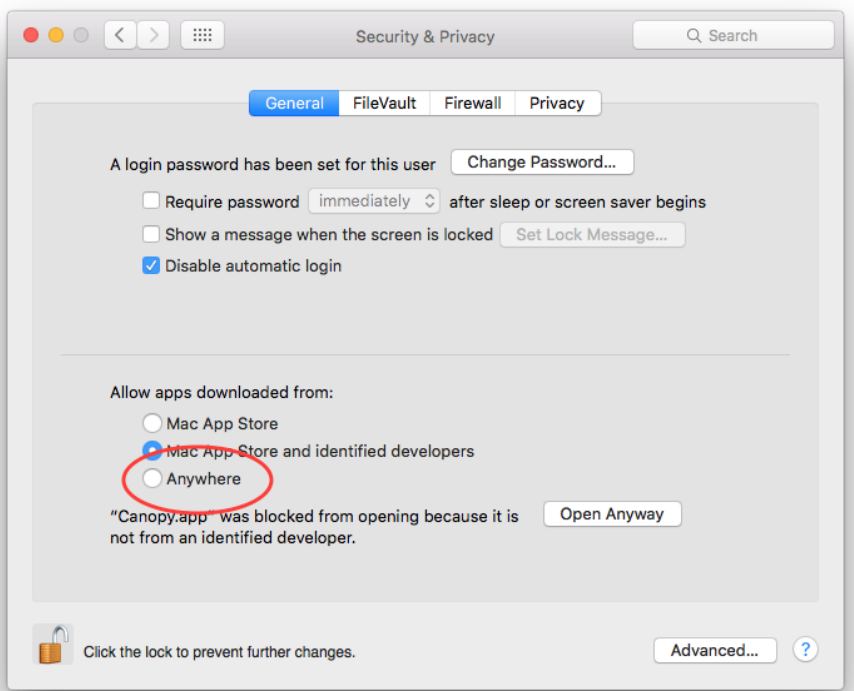

Re-launch Canopy.app from Finder. After Canopy runs, you may restore your original Allow apps downloaded from settings.

\subsection{Step 3: Install the h5py Module}

From the Canopy application, launch the Package Manager by clicking on the icon.

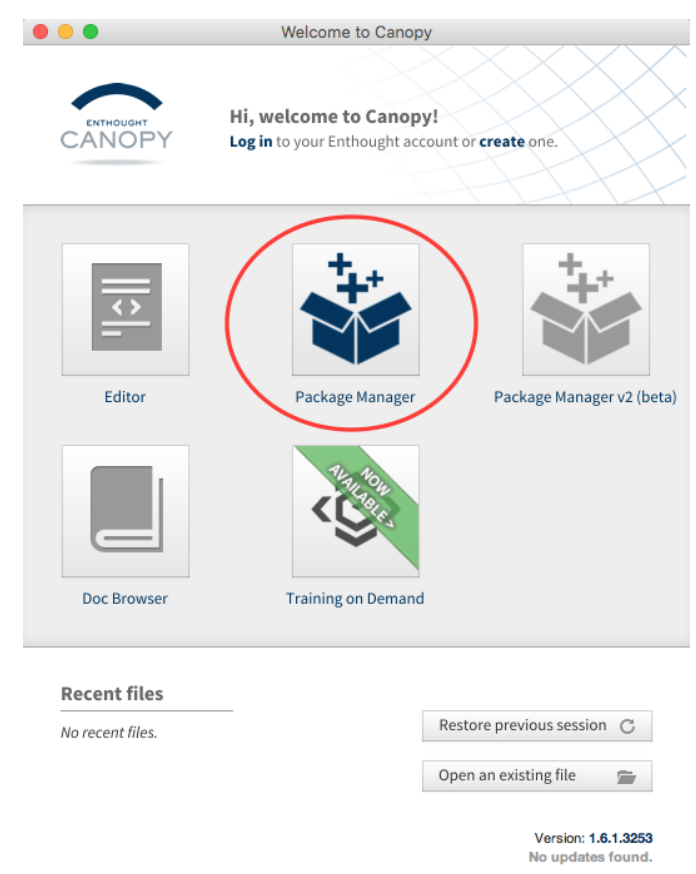

With Available Packages selected, enter "h5py" in the search box. The package named "h5py 2.6.0-2" will appear in the package list. 


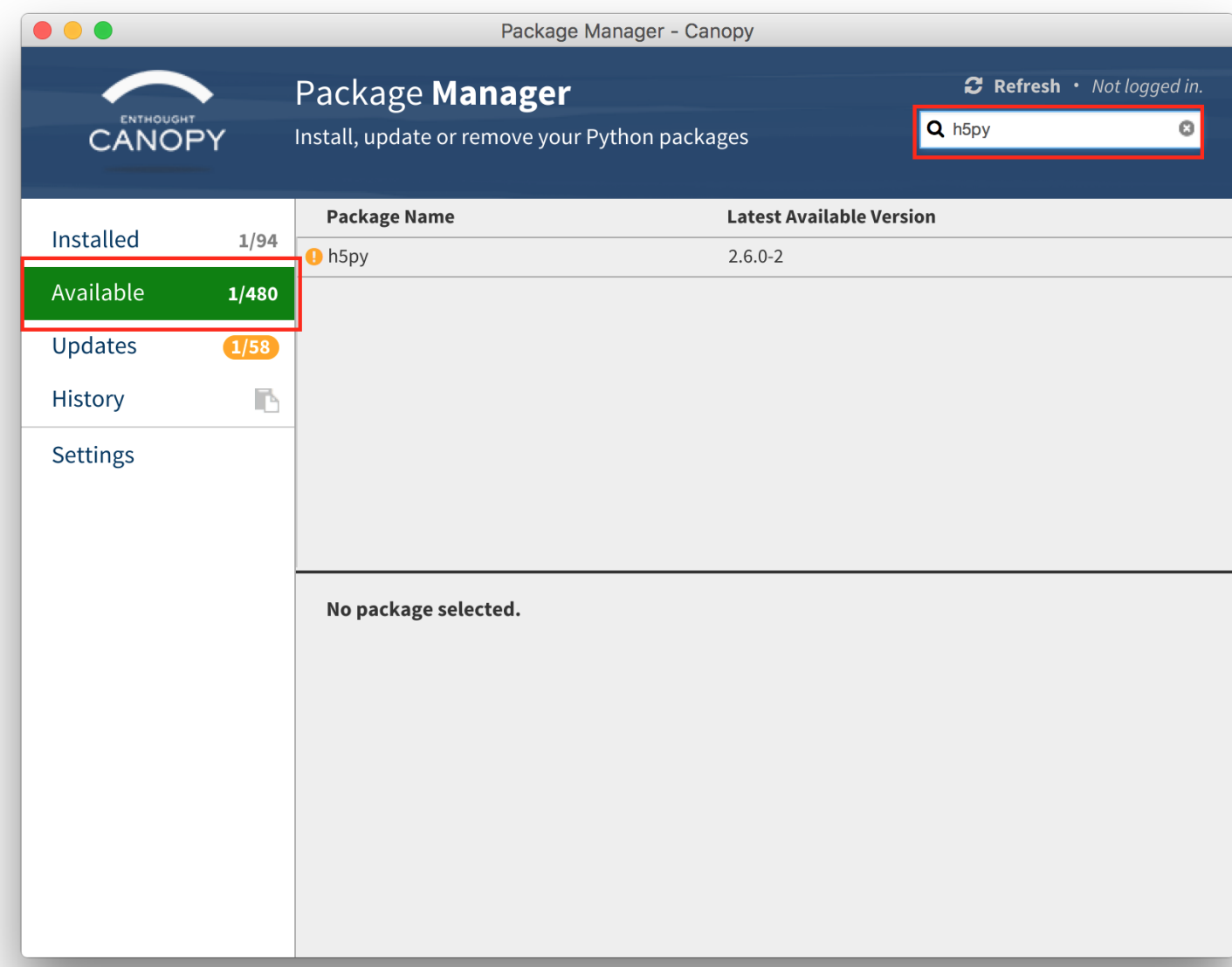

Click h5py 2.6.0-2 to select it. Activate the Install (or Upgrade to) button to install it.

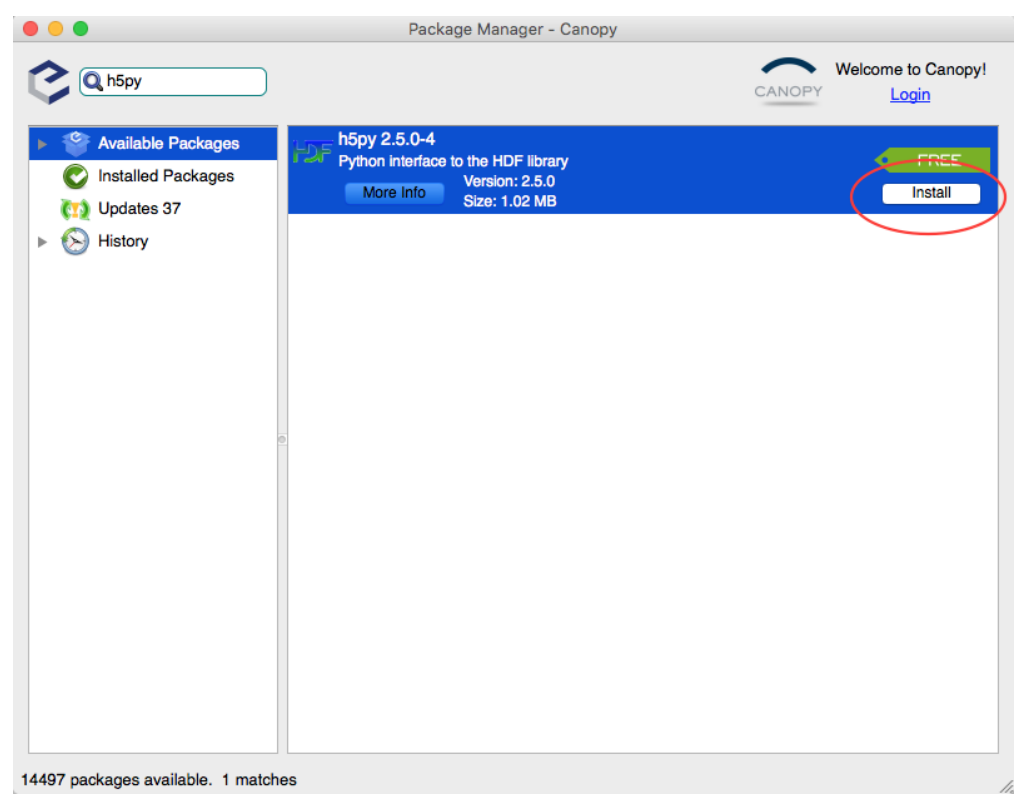


The module and dependency eggs will be downloaded and installed. When complete, the Install button will be renamed to Uninstall.

\subsection{Step 4: Install the wxPython-3.0.2 Module}

As per Step 3, seach for "wxPython" in Available Packages. This will result in wxPython 3.0.2.0-3 appearing in the list panel. Click wxPython 3.0.2.0-3 and activate the Install (or Upgrade to) button to install wxPython and all its dependencies.

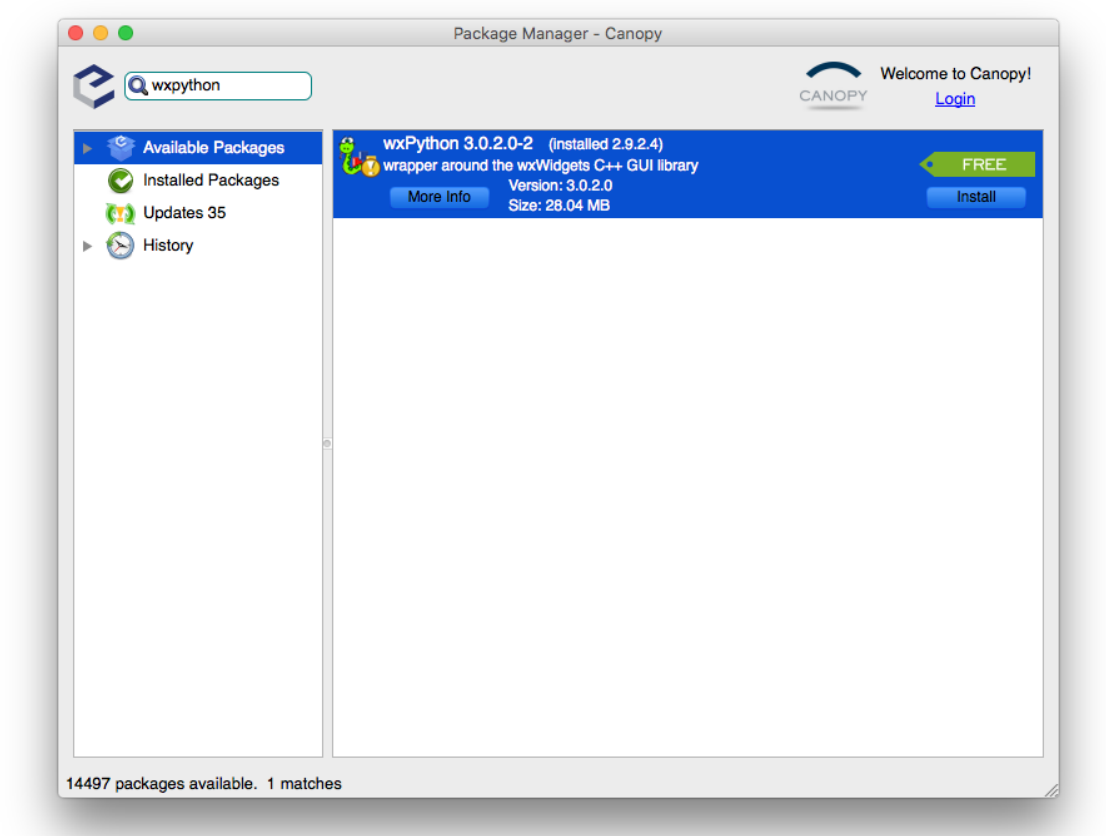

Note:

If VERAView fails with a message about image formats, you should downgrade to wxPython 3.0.2.0-01 by selected that version from the Available combo box and activating the Downgrade to v3.0.2.0-1 button.

\subsection{Step 5: Install the mayava-4.4.3 Module}

As per Steps 3 and 4, search for "mayavi" in Available Packages. This will result in mayavi 4.4.3-10 appearing in the panel. Click mayavi 4.4.3-10 and activate the Install (or Upgrade to) button. 


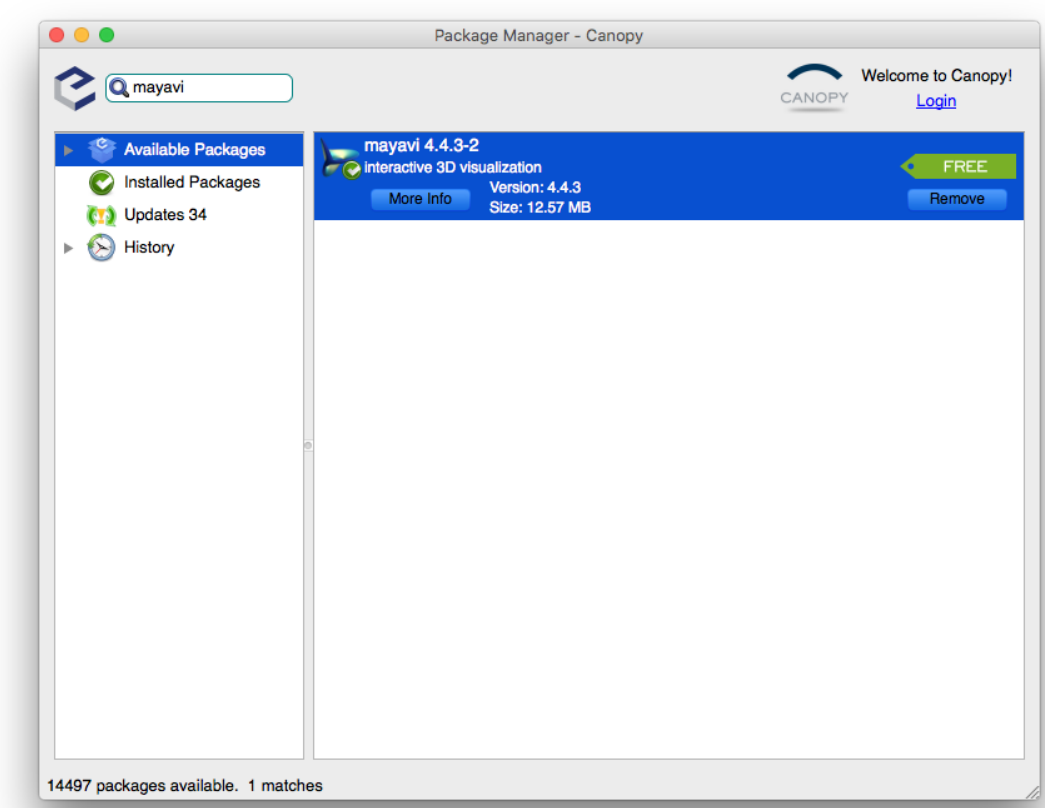

\subsection{Step 6: Test the Environment}

This is an optional but recommended step that involves launching a Terminal window and running Python from a shell. If you installed to the default location, the path to Python will be as shown below.:

\$ /Library/Enthought/Canopy_64bit/User/bin/python

Enthought Canopy Python 2.7.11| 64-bit | (default, Jun 11 2016, 03:41:56)

[GCC 4.2.1 Compatible Apple LLVM 6.0 (clang-600.0.57)] on darwin

Type "help", "copyright", "credits" or "license" for more information. $>>>$

Download the following script to test that needed modules are available.:

https://casl:rocksanewton.ornl.gov/ re7/xfer/casl/test/test-install.py

Run the test script from the command line.:

\$ /Library/Enthought/Canopy_64bit/User/bin/python test-install.py

Importing h5py and wx...

wx.version= 3.0.2.0 osx-cocoa (classic)

Importing numpy...

Importing mayavi...

Good to go! 


\section{Linux}

\subsection{Step 1: Install Canopy}

Download canopy-1.7.4-rh5-64.sh from the Enthought site or locally:

https://store.enthought.com/downloads/\#default

Note you can download a 32-bit version from Enthought if needed.

Note this Canopy environment is built on Red Hat 5 and thus should be binary compatible with recent versions of most Linux distributions. Also, this is a per-user install that does not require root privileges.

Execute the installer by running canopy-1.7.4-rh5-64.sh with a bash shell. When prompted to review the license type $<$ Enter $>$ :

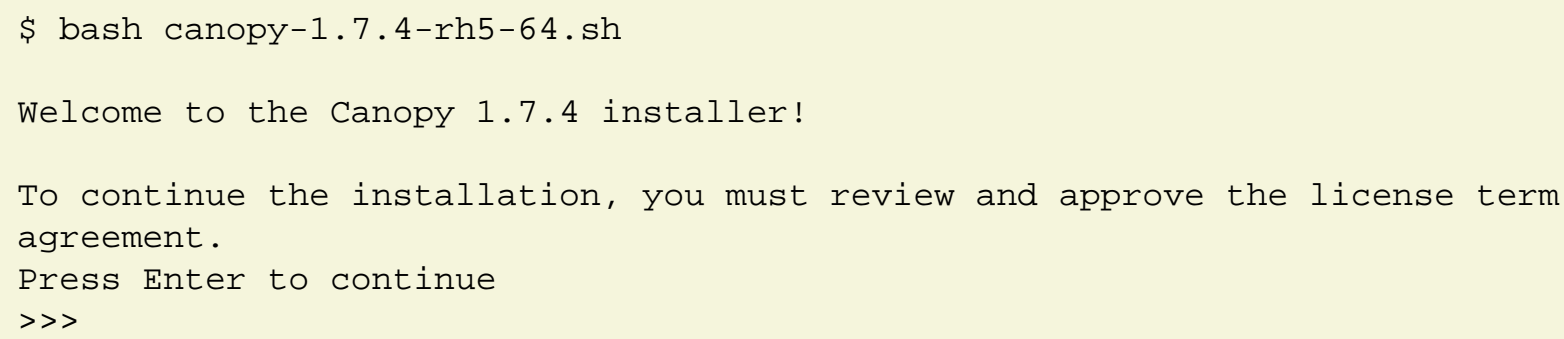

Press $<$ Space $>>$ to view each license page. When all pages have been viewed, you will be prompted to approve the license. Type "yes" and <Enter $>$ to continue.:

Do you approve the license terms? [yes|no]

[no] $>>>$ yes

Next, you will be prompted for the directory in which to install Canopy. The default will be a Canopy subdir in your home directory. Accept this default by typing $<$ Enter $>$ :

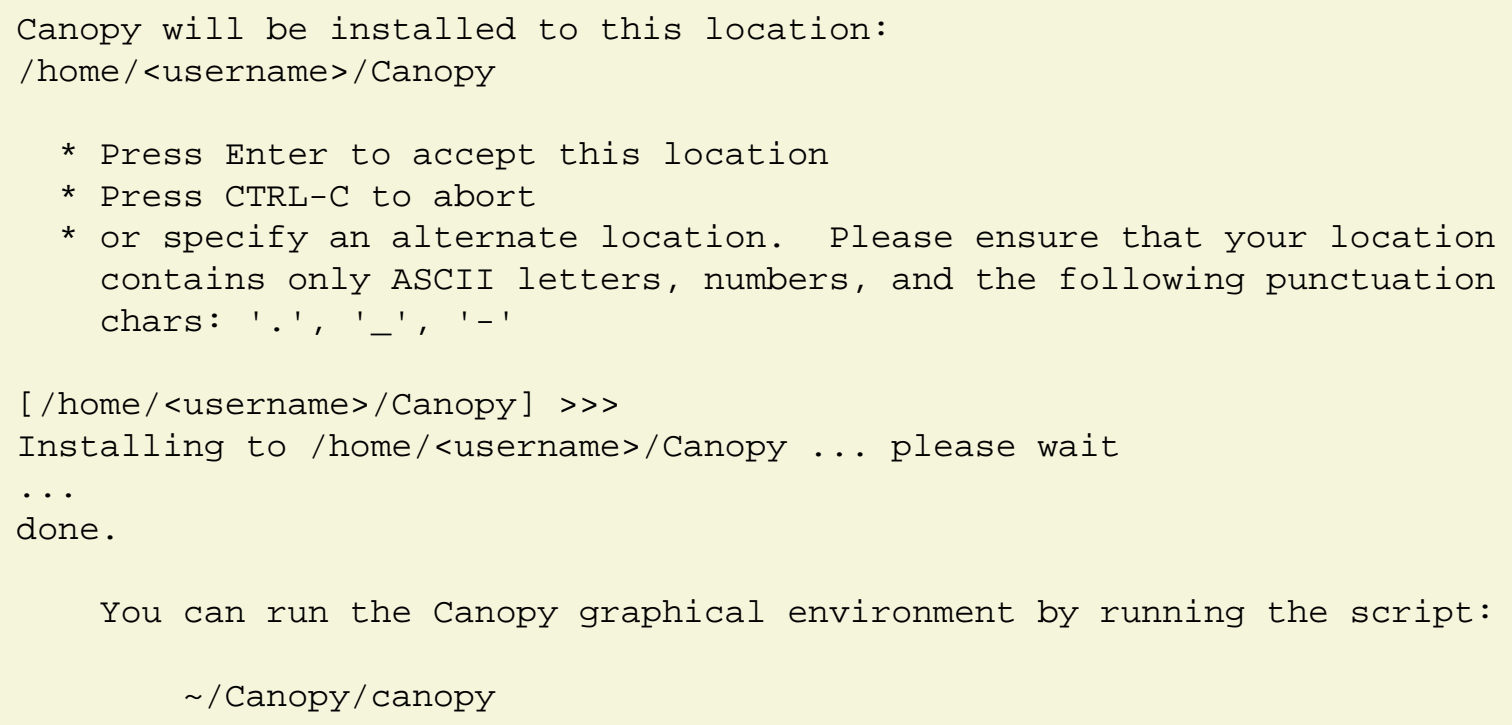


or by selecting 'Canopy' in your Applications menu.

On your first run, your Canopy User Python environment will be initialized, and you will have the opportunity to make Canopy be your default Python

at the command line. Details at support.enthought.com/forums

Thank you for installing Canopy!

\subsection{Step 2: Setup the Canopy Environment}

Execute the Canopy GUI application to set up the environmet for your user account by executing the path shown.:

\$ $~ / C a n o p y / c a n o p y$

By default, Canopy will install to an Enthought subdirectory in your home directory, \$HOME/Enthought/Canopy_64bit. If you change this location, you will need to manually edit the veraview-linux.run.sh file inside your VERAView installation directory. Unless you have good reason to choose an alternate location (via the Change button), accept the default directory and activate the Continue button.

\begin{tabular}{|l|}
\hline \multicolumn{1}{|c|}{ Canopy Environment Setup } \\
Canopy System and User environment locations \\
Your Canopy environment will be installed in the location shown below. \\
You may change it, if you wish to. What's this \\
Canopy environment directory \\
/home/re7/Enthought/Canopy_64bit \\
\hline
\end{tabular}

After a few seconds, a progress dialog will appear. It can take several minutes for the setup process to complete.

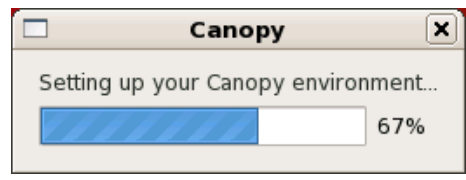

When the setup completes, you will be prompted to make Canopy's Python your default Python environment. This is completely up to you, for the VERAView launch script will reference Canopy directly. Activate the Starting using Canopy button to launch the Canopy application. 
Make Canopy your default Python environment?

Do you want to make Canopy your default Python environment?

- Yes (Recommended)

This will give you direct access to Canopy Python, and to utilities like IPython, easy_install, nosetests, from your terminal / command prompt. Learn More

No

Later on, if you want to make Canopy Python the default, you can do so from the

preferences dialog. Warning - If you plan to manually specify the full path to

Canopy Python, you must specify Canopy's "User" Python, rather than the Canopy installation Python. Learn More

\section{Start using Canopy}

\subsection{Step 3: Install the h5py Module}

From the Canopy application, launch the Package Manager by clicking on the icon.

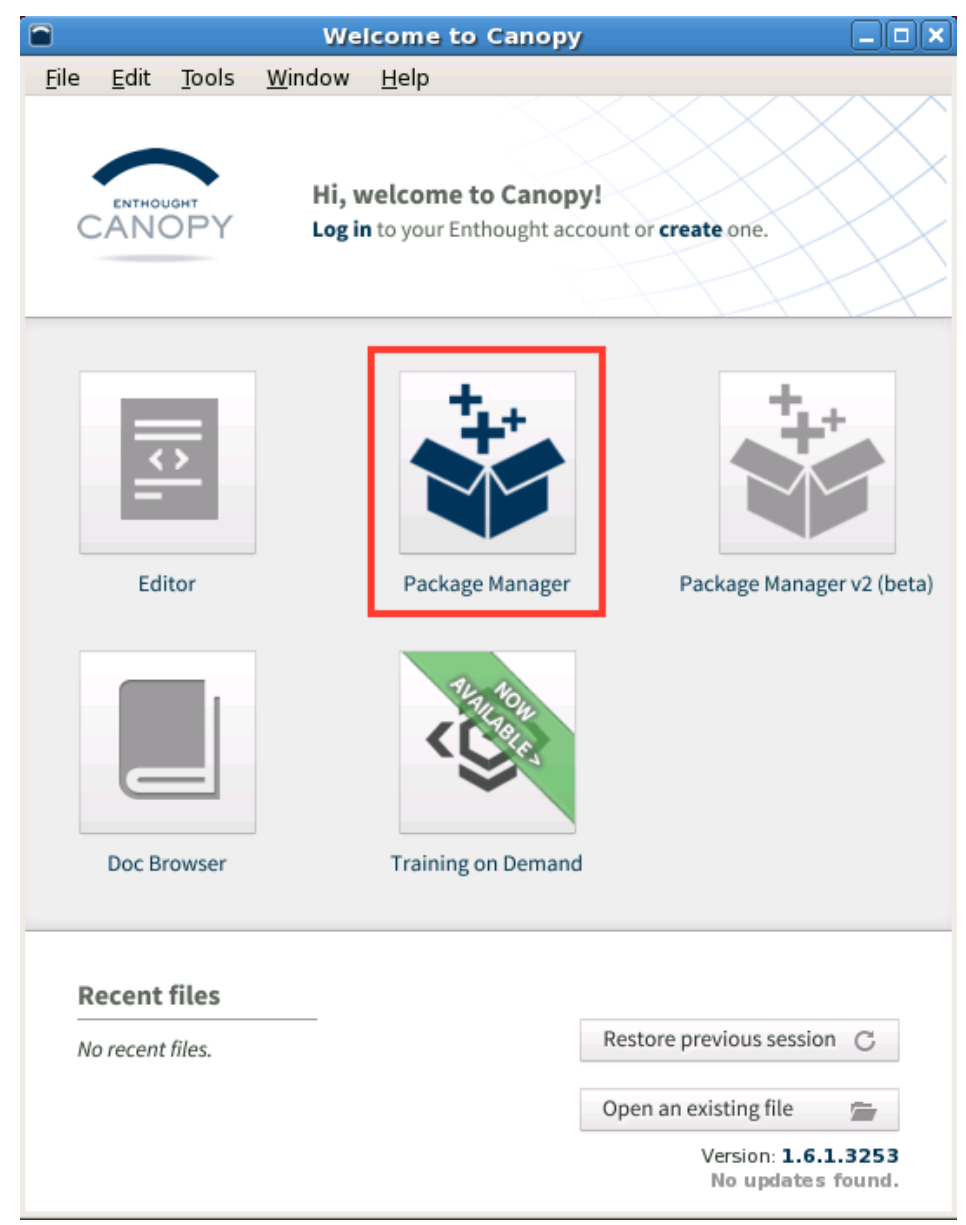

With Available Packages selected, enter "h5py" in the search box. The package named "h5py 2.6.0-2" will appear in the package list panel. 
Install Canopy for VERAView, 2016-09-12

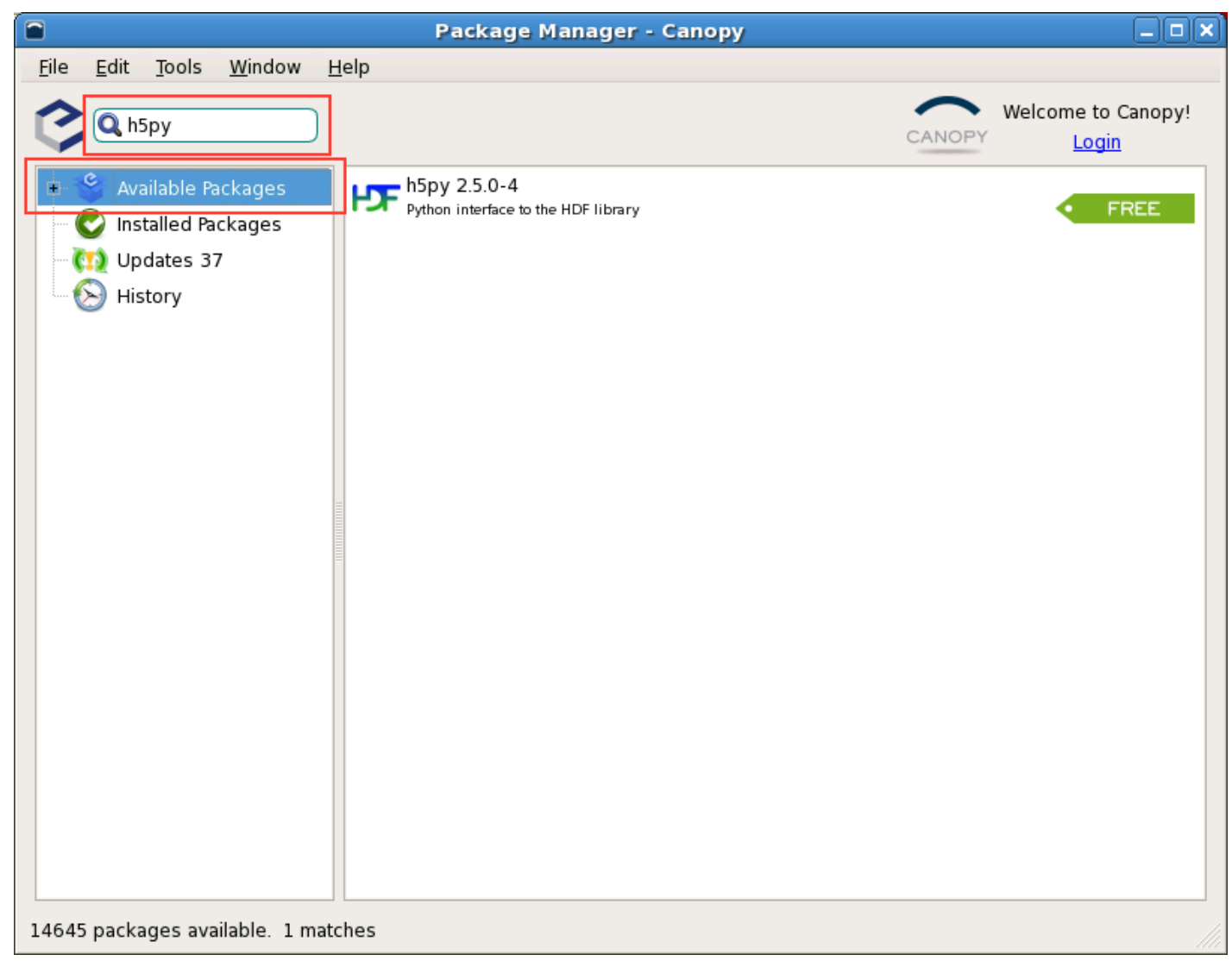

Click $h 5 p y$ 2.6.0-2 in the list panel to select it. Activate the Install (or Upgrade to) button to install it. 
Install Canopy for VERAView, 2016-09-12

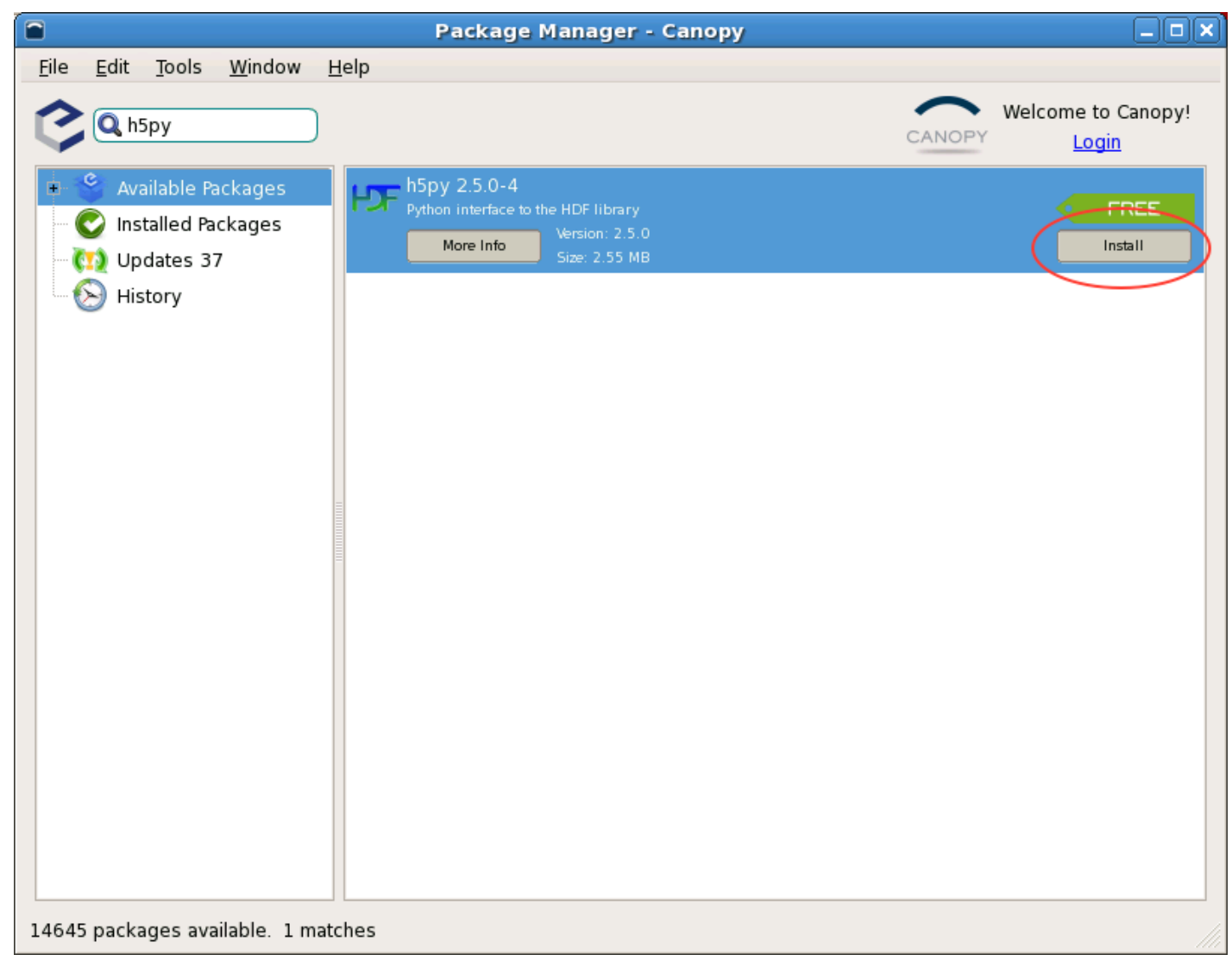

The module and dependency eggs will be downloaded and installed. When complete, the Install button will be renamed to Remove. 


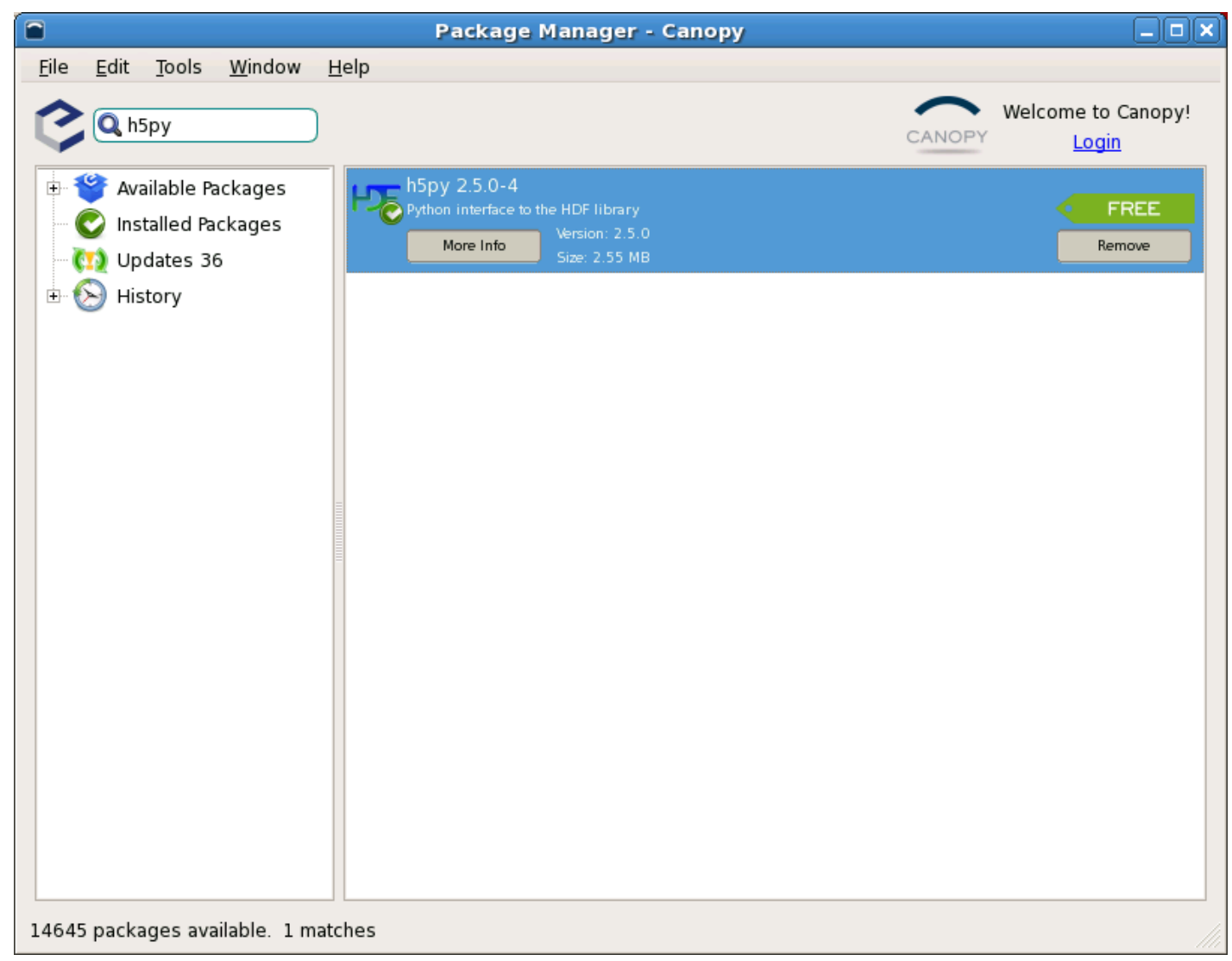

\subsection{Step 4: Install the wxPython-3.0.2 Module}

As per Step 3, seach for "wxPython" in Available Packages. This will result in wxPython 3.0.2.0-3 appearing in the list panel. Click wxPython 3.0.2.0-3 and activate the Install button to install wxPython and all its dependencies. 


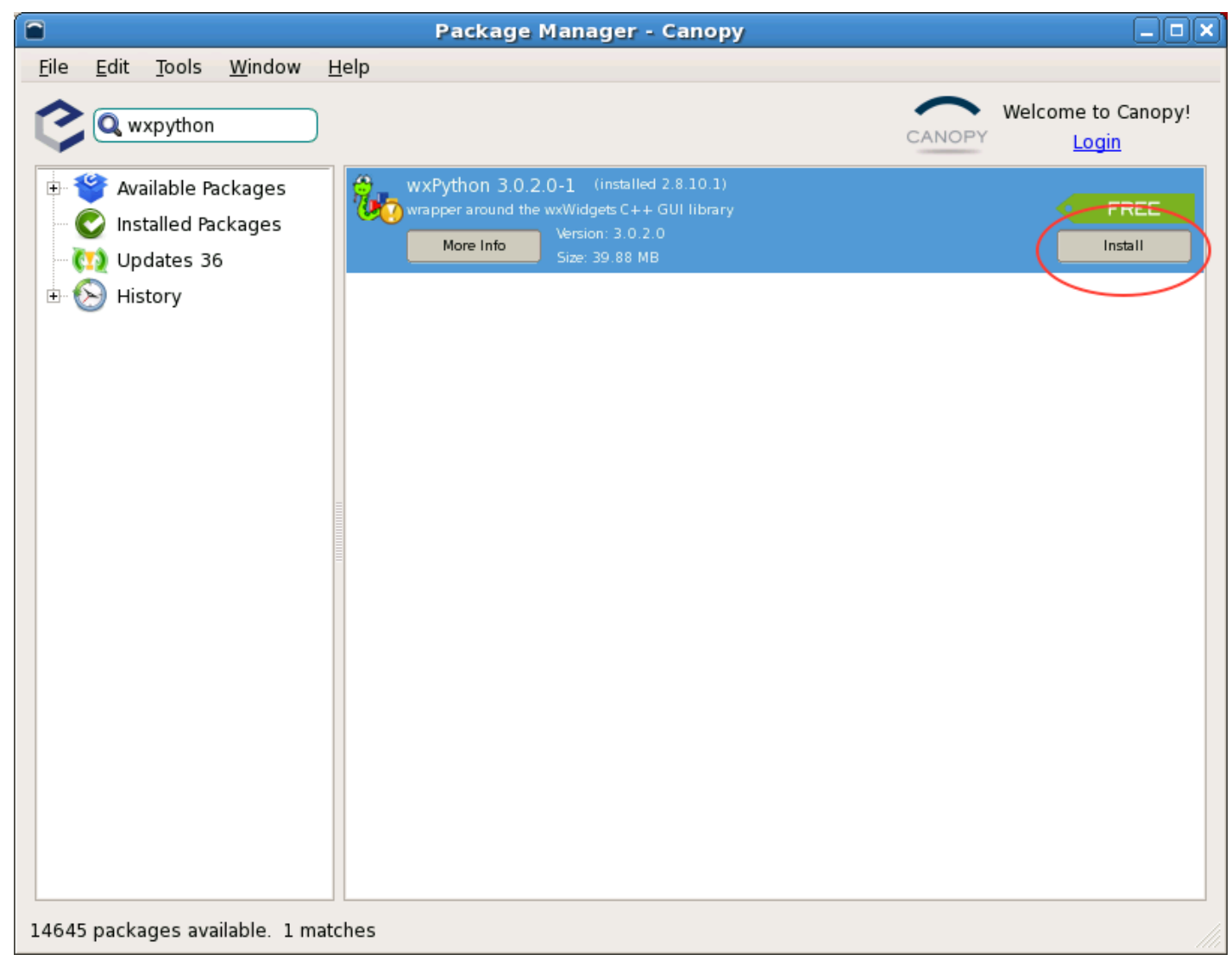

\section{Note:}

If VERAView fails with a message about image formats, you should downgrade to wxPython 3.0.2.0-1 by selected that version from the Available combo box and activating the Downgrade to v3.0.2.0-1 button.

\subsection{Step 5: Install the mayava-4.4.3-2 Module}

As per Steps 3 and 4, search for "mayavi" in Available Packages. This will result in mayavi 4.4.3-10 appearing in the list panel. Click mayavi 4.4.3-10 and activate the Install button. 


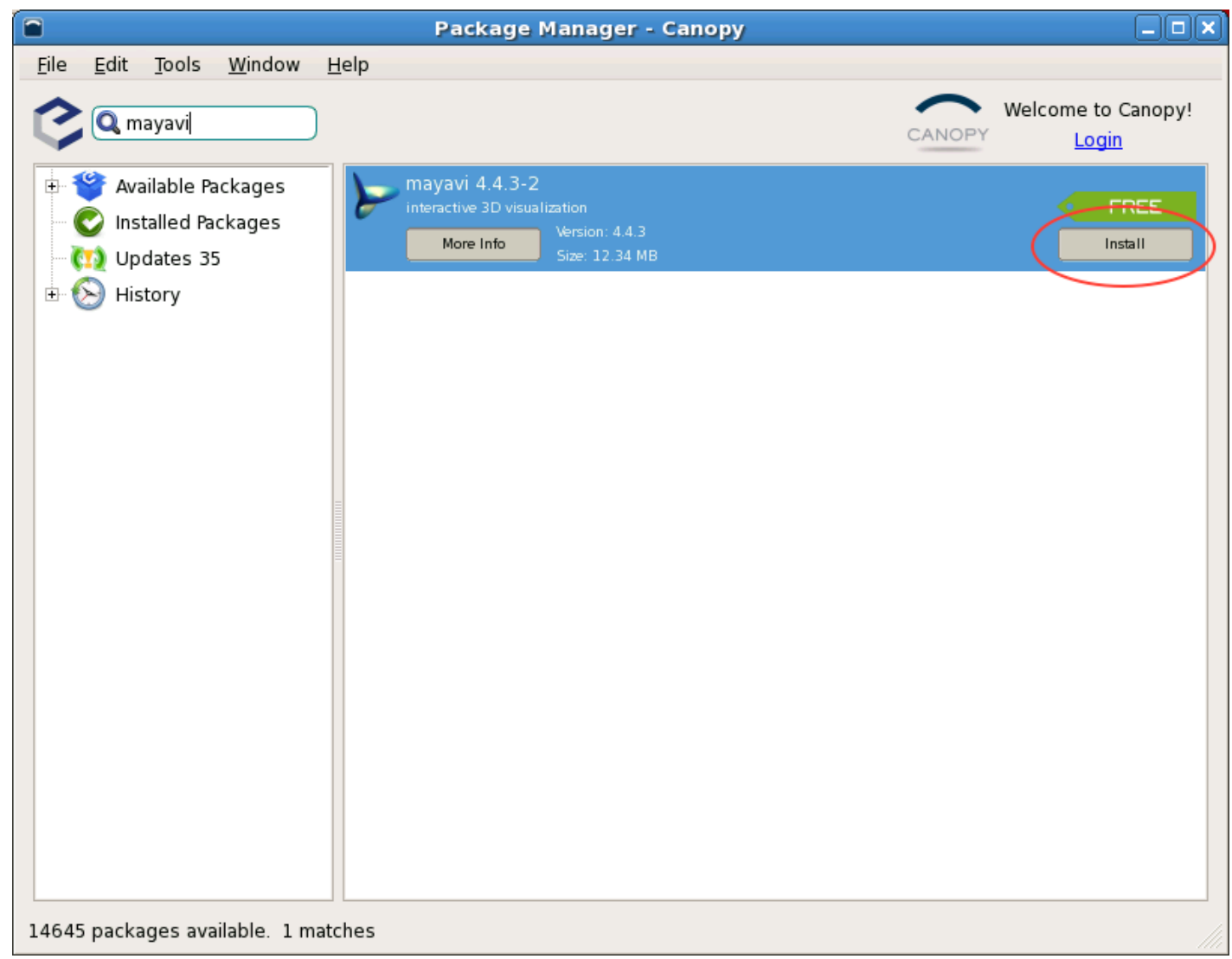

\subsection{Step 6: Test the Environment}

This is an optional but recommended step that involves launching a Python shell and interactively entering import statements. Run the Python shell by entering the path to the Python executable in a bash shell. If you installed to the default location, the path will be as shown below.:

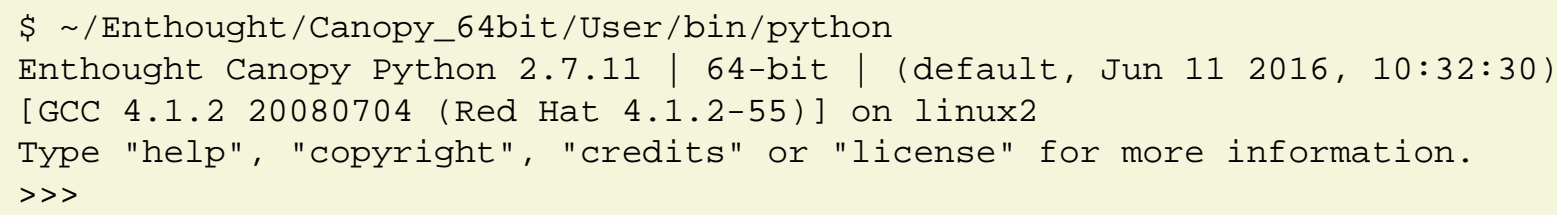

Download the following script to test that needed modules are available.:

https://casl:rocksenewton.ornl.gov/ re7/xfer/casl/test/test-install.py

Run the test script from the command line.:

\$ /Enthought/Canopy_64bit/User/bin/python test-install.py

Importing h5py and wx...

wx.version $=3.0 .2 .0$ gtk2 (classic)

Importing numpy...

Importing mayavi... 
Good to go!

Note:

There are many Linux distributions for which the Linux Canopy install will just not work. We will address this in a future version with a Docker image that can be run on most distros. 